\title{
Synthesis and Characterization of Furans and Levulinates Polymers: Derived from Cellulosic Carbohydrates via Aldol Condensation
}

\author{
Jin Tan ${ }^{1,2,3}$, Chenguang Wang ${ }^{1,2,3}$, Qi Zhang ${ }^{1,2,3}$ and Longlong $\mathrm{Ma}^{1,2,3, *}$ \\ ${ }^{1}$ Guangzhou Institute of Energy Conversion, Chinese Academy of Sciences (CAS), Guangzhou, Guangdong, China \\ ${ }^{2}$ CAS Key Laboratory of Renewable Energy, Guangzhou, Guangdong, China \\ ${ }^{3}$ Guangdong Provincial Key Laboratory of New and Renewable Energy Research and Development, Guangzhou, Guangdong, China
}

\begin{abstract}
Aldol condensation between furans and levulinates shows a selective route for the polymers production. In this work, the influences of mole ratio between furans and levulinates as well as substrate species on the formation of polymers structure were investigated. The characterization of gel permeation chromatography and ${ }^{13} \mathrm{C}$ nuclear magnetic resonance to the synthesized polymers suggested that the mole ratio between furans and levulinates, and substrate species exhibited a great effect on the structure of polymers. Higher mole ratios between furans and levulinates resulted in the aggravated polymerization. EMF-EL polymers showed larger molecular weight distribution compared with FFA-EL/LA polymers. This investigation further provides other key factors to the decision of polymer structure.
\end{abstract}

\section{Introduction}

Serious energy crisis and worse environment problem compel human to explore a renewable green fuels to substitute the traditional fossil fuels [1]. As an important kind of platform chemicals derived from biomass, furans and levulinates have extensive advantages in synthesis of biofuels with different carbon chains or conversion to blending component in biodiesel [2]. C-C coupling reaction between furans and levulinates is an effective method in the synthesis of long-chained biofuels via aldol condensation [3-5].

In the synthetic process of long-chained biofuels from biomass, furfural (FFA), hydroxymethylfurfural (HMF) and 5-ethoxymethylfurfural (EMF) are widely used furanic materials, and ethyl levulinate (EL) and levulinic acid (LA) are typically adopted levulinates chemicals [3, 6]. It is well known that cellulose, hemicellulose and lignin mainly consist of biomass, in which cellulose fractions are identified as the most abundant renewable materials in nature world. Commonly, the synthetic routes for furans and levulinates production from biomass are as follows: FFA is commercially produced from the successive dehydration of hemicellulose over acid catalysts [7]. While production of FFA from cellulose is technologically challenging. EL comes from esterification of LA in ethanol, promoted by acid catalysts. LA is produced from the rehydration of HMF following cellulose dehydration by acid catalysts [8]. Because of the active hydroxy groups in HMF, it is usually etherified to EMF in ethanol to reduce the risk in polymerization [9].
Nowadays, those substrates used in the synthesis of long-chained biofuels still depend and require the separated and purified furans and levulinates [10]. Although they can be obtained from biomass through alcoholysis/hydrolysis with higher yields, it is not economical to recover those versatile platform chemicals after alcoholysis/hydrolysis of biomass. Moreover, utilization of the abundant cellulose fractions in biomass will enrich the feedstock source in producing FFA and HMF. Therefore, it is meaningful to synthesize longchained biofuels adopting cellulosic carbohydrates which derived from cellulose fractions with no separation and purification procedures. However, it remains a continuous challenge to bring about $\mathrm{C}-\mathrm{C}$ bond formation with good activity and selectivity. According to the previous literature [3], different substrates before reaction give different structures of condensation polymers by selecting aldol condensation or self-Michael additions. Reaction time seems to be an another key factor in affecting condensation mechanism [3]. Furthermore, our previous investigation show that the stoichiometric ratios between furans and levulinates are related to the formation of polymers structure [6].

In this work, the influences of substrates species as well as the stoichiometric ratios between furans and levulinates on the structures of polymers were further investigated. Meanwhile, the characterization for polymers was performed by gel permeation chromatography (GPC) and ${ }^{13} \mathrm{C}$ nuclear magnetic resonance $\left({ }^{13} \mathrm{C}\right.$ NMR) to identify the structure of polymers.

\footnotetext{
* Corresponding author: mall@ms.giec.ac.cn
} 


\section{Materials and methods}

\subsection{Materials}

EMF and EL came from the alcoholysis of fructose in pure ethanol, and EL and LA were produced from cassava depolymerization in ethanol-water medium. $\mathrm{H}_{2} \mathrm{SO}_{4}$, and $\mathrm{NaOH}$ were all obtained from Damao Chemical Reagents Company, China. $\mathrm{C}_{2} \mathrm{H}_{5} \mathrm{OH}$ was bought from Guangdong Guanghua Sci-Tech Co., Ltd., China. FFA was purchased from Aladdin Reagent Company.

\subsection{Polymers production}

The mixtures of furans and levulinates at suitable stoichiometric ratios for polymers production were introduced to a $100 \mathrm{~mL}$ stainless-steel reactor with a Teflon interior. The sealed reactor was kept at $373 \mathrm{~K}$ for $4.0 \mathrm{~h}$ and the system was halted and cooled down to room temperature by cold water. Then ethanol was removed completely from reaction system by rotary evaporation following water addition to ensure an absolute water medium. Finally, polymers were in the form of solid after adding sulfuric acid and they were separated from solution by filtration. The detailed steps for pure polymers production from fructose and cassava are exhibited in Fig. 1.

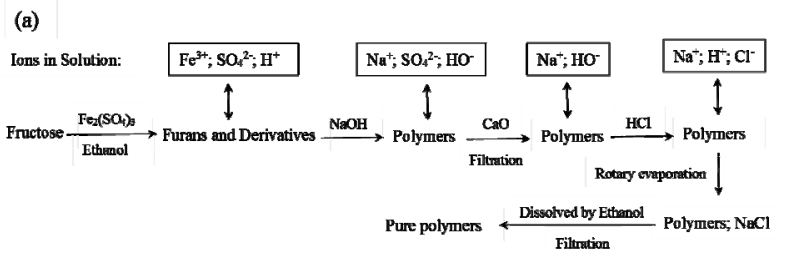

(b)

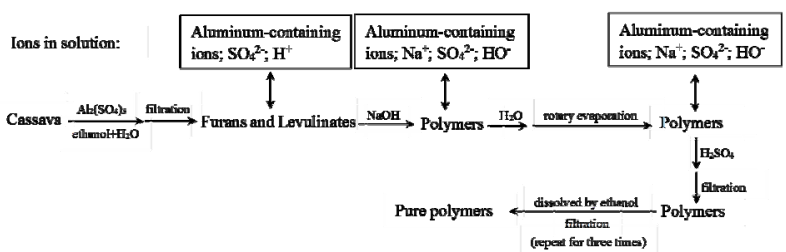

Fig. 1. Scheme of the achieved pure polymers from fructose (a) and cassava (b).

\subsection{Analytical methods}

The average molecular weight of polymers were evaluated through GPC-1260 Infinity (Agilent PLgel 3 $\mu \mathrm{m}$ MIXED, tetrahydrofuran, $1.0 \mathrm{~mL} / \mathrm{min}, 298 \mathrm{~K}$ ). Meanwhile, ${ }^{13} \mathrm{C}$ chemical shifts of polymers were measured using a NMR scanner (Bruker Advance 400 III) with dimethyl sulfoxide (DMSO) as the solvent (100 $\mathrm{MHz}$ ).

\section{Results and Discussion}

\subsection{Aldol condensation pathways between furans and levulinates}

The pathways for furans and levulinates polymer production via aldol condensation are shown in Fig. 2. Aldol condensation between furans and levulinates is a typical base catalyzed process by coupling of the carbon atoms having $\alpha-\mathrm{H}$ in levulinates and the carbonyl group in furfural via carbanion mechanism $[11,12]$. there are at most two carbon active sites in furan derivatives formed under alkaline conditions. Therefore, only one or two aldehyde carbons of furans can be interlinked with active carbons via $\mathrm{C}-\mathrm{C}$ coupling [3, 13]. According to our previous investigations $[6,14], \mathrm{EL}$ and LA are the final products after cassava depolymerization. To ensure the stoichiometric ratios between furans and levulinates are at $1: 1$ and $2: 1$, extra FFA are added into the condensed system directly based on the mole content of levulinates. Moreover, to avoid adding extra furans before condensation, the mole ratios between furans and levulinates are adjusted in the range of 1 to 2 by optimizing reaction parameters and catalysts in the process of fructose alcoholysis. Finally, EMF and EL are the main components after fructose alcoholysis, and the stoichiometric ratios between EMF and EL are at $1.1: 1$ and $1.9: 1$ respectively.
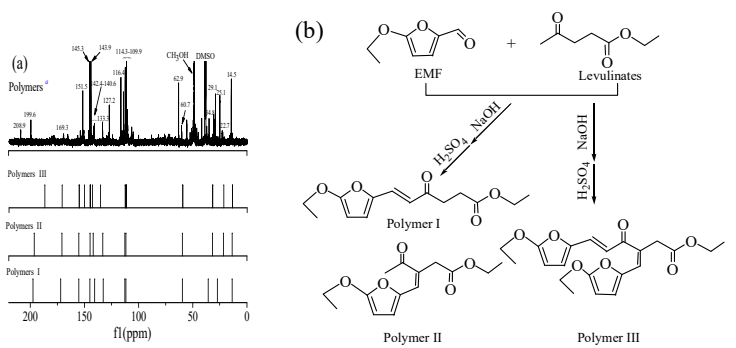

Fig. 2. Proposed main pathways of aldol condensation between furans and levulinates. (a) FFA and levulinates, (b) EMF and EL

\subsection{GPC characterization for condensation polymers}

Previous investigation reveals that the polymer structures are closely connected with the reaction parameters during condensation, such as reaction time and substrates species [3]. In this work, FFA-EL/LA polymers and EMF-EL polymers were synthesized at different stoichiometric ratios. Fig. 3 and Fig. 4 show the GPC characterization results of condensation polymers. It is obvious to see that a wide-spreading average molecular weight can be recognized, regardless of the different substrate species (EMF-EL or FFA-EL/LA) and mole ratios between furans and levulinates $(1: 1$ or $2: 1)$.
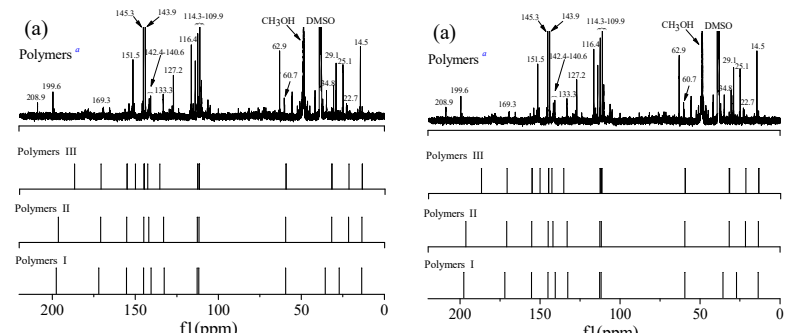

Fig. 3. GPC characterization for the synthesized FFA-EL/LA polymers. Mole ratio: (a) $1: 1$ and (b) $2: 1$. 

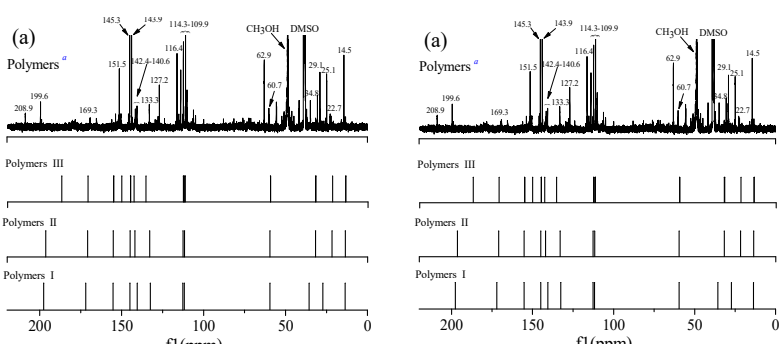

Fig. 4. GPC characterization for the synthesized EMF-EL polymers. Mole ratio: (a) $1.1: 1$; (b) $1.9: 1$.

Because of the different condensation pathways between furans and levulinates, theoretical polymer I, II, and III can be formed simultaneously in the same reaction system (Fig. 2), which results in the widespreading average molecular weight. Meanwhile, an increase in the possibility of the polymerization reaction through changing the mole ratio increase the average molecular weight. Hence, the carbon chains are prolonged through aldol condensation and self-Michael addition, resulting in the complex mixture of polymers. Furthermore, EMF-EL polymers produced at the mole ratio of $1.9: 1$ show larger average molecular weights compared with FFA-EL/LA polymers produced at the mole ratio of $2: 1$. This result indicates that condensation reaction at higher mole ratios between EMF and EL are apt to form larger molecular weight polymers compared with FFA-EL/LA.

\section{3 ${ }^{13} \mathrm{C}$ NMR characterization for condensation polymers}

The synthesized polymer is a mixture of compounds, and this conclusion is further supported by ${ }^{13} \mathrm{C}$ NMR (Fig. 5 and Fig. 6). The compared results of ${ }^{13} \mathrm{C}$ NMR between FFA-EL/LA polymers (produced at the mole ratio of 1 : 1 and $2: 1$ ) and theoretical FFA-EL polymers (produced at the mole ratio of $1: 1$ and $2: 1$ ) show that a majority of chemical shifts of the synthesized polymers are in accordance with those of the theoretical polymers (polymer I, II, and III) (Fig. 5). EMF-EL polymers show the same molecular weight distribution characteristic as FFA-EL/LA polymers. However, the degree of polymerizations is seem to be enhanced at higher mole ratio. Especially when EMF and EL are adopted as substrates for condensation (Fig. 6).
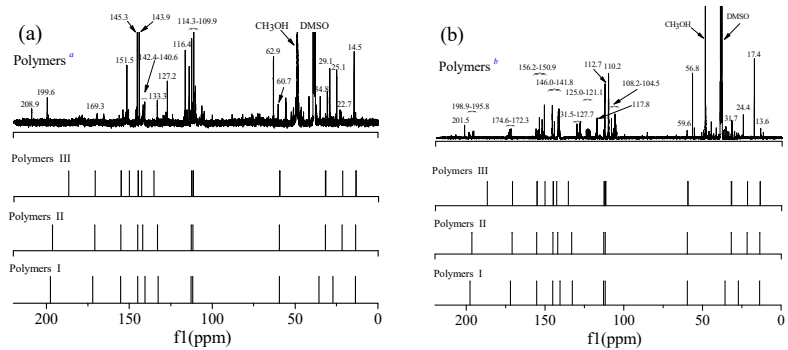

Fig. 5. ${ }^{13} \mathrm{C}$ NMR comparison of the synthesized FFA-EL/LA polymers with theoretical FFA-EL polymers. Mole ratio: (a) 1 : 1 , (b) $2: 1$.
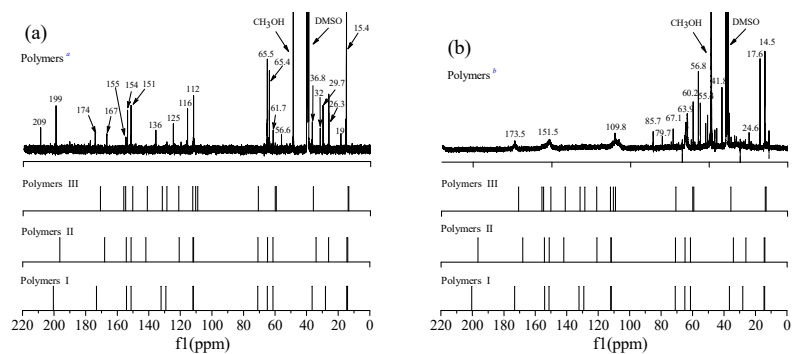

Fig. 6. ${ }^{13} \mathrm{C}$ NMR comparison of the synthesized EMF-EL polymers with theoretical EMF-EL polymers. Mole ratio: (a) $1.1: 1$, (b) $1.9: 1$.

Therefore, we can conclude that the mole ratio and substrate species affect the condensation mechanism and control the length of the carbon chains of the polymers by aldol condensation and Michael additions. Therefore, those undefined chemical shifts are probably attributed to the other condensation products.

\section{Conclusions}

Condensation polymers can be synthesized from furans and levulinates which derived from renewable cellulosic carbohydrates. The mole ratios between furans and levulinates as well as substrate species give great effect on the structure of polymers. Those substrates with higher mole ratios result in the aggravating polymerization, and EMF-EL polymers show larger molecular weight distribution compared with FFAEL/LA polymers.

\section{Acknowledgments}

This work was supported by the National Natural Science Foundation of China (No. 51536009), Local Innovative and Research Teams Project of Guangdong Pearl River Talents Program (No. 2017BT01N092) and Strategic Priority Research Program of the Chinese Academy of Sciences (No. XDA21060102).

\section{References}

1. C.J. Chuck, J. Donnelly, Appl. Energ. 118(2014), 83-91.

2. L. Peng, L. Lin, J. Zhang, J. Shi, S. Liu, Appl. Catal. A-Gen. 397(2011), 259-265.

3. A.S. Amarasekara, T.B. Singh, E. Larkin, M.A. Hasan, H.J. Fan, Ind. Crop. Prod. 65(2015), 546-549.

4. C. Li, D. Ding, Q. Xia, X. Liu, Y. Wang, ChemSusChem 9(2016),1712-1718.

5. G. Liang, A. Wang, X. Zhao, N. Lei, T. Zhang, Green Chem. 18(2016), 3430-3438.

6. J. Tan, Q. Liu, C. Cai, S. Qiu, T. Wang, Q. Zhang, L. Ma, G. Chen, RSC Adv. 5(2015), 58784-58789.

7. K. Yoshida, H. Nanao, Y. Kiyozumi, K. Sato, O. Sato, A. Yamaguchi, M. Shirai, J. Taiwan Inst. Chem. Eng. 79(2017), 55-59. 
8. W. Zhao, Y. Li, C. Song, S. Liu, X. Li, J. Long, Appl. Energ. 204(2017), 1094-1100.

9. X. Hu, C. Lievens, A. Larcher, C.Z. Li, Bioresour. Technol. 102(2011), 10104-10113.

10. K. Yan, Y. Liu, Y. Lu, J. Chai, L. Sun, Catal. Sci. Technol. 7(2017), 1622-1645.

11. L. Faba, E. Diaz, S. Ordonez, ChemSusChem 7(2014), 2816-2820.

12. C. Cai, Q. Liu, J. Tan, T. Wang, Q. Zhang, L. Ma, Korean Chem. Eng. Res. 54(2016), 1-8.

13. J.P. Lange, E. van der Heide, J. van Buijtenen, R. Price, ChemSusChem 5(2012), 150-166.

14. J. Tan, Q. Liu, L. Chen, T. Wang, L. Ma, G. Chen, J. Energ. Chem. 26(2017), 115-120. 